ESAIM: PROCEEDINGS, December 2008, Vol. 25, p. 80-90

E. Cancès, S. Faure, B. Graille, Editors

\title{
SIMULATION OF THE BREAK-UP OF DIPHASIC JETS IN A MICROCHANNEL
}

\author{
Charles-Henri Bruneau $^{1},{\text { Thierry } \text { Colin }^{2} \text { and Tancogne Sandra }}^{3}$
}

\begin{abstract}
In this paper, diphasic flows driven by pressure gradient in channels of a few hundred of micrometres square sections are studied. At this scale, the interfacial effects are dominating and the instabilities processes are generally studied from the experimental point of view. The instability is a well-known hydrodynamic instability due to surface tension namely the Plateau-Rayleigh instability. In this work, tridimensional numerical simulations are presented to understand this phenomenon and more generally to study the behaviour of diphasic fluids evolving in square microchannels.
\end{abstract}

Résumé. Dans ce papier, des écoulements diphasiques évoluant dans des canaux possédant des sections carrées de l'ordre de la centaine de micromètres sont étudiés. A cette échelle, les effets interfaciaux sont prépondérants et les mécanismes d'instabilités de jets de fluides y sont généralement étudiés du point de vue expérimental. L'instabilité sous-jacente est l'instabilité de Plateau-Rayleigh. Son mécanisme est ici analysé grâce à la simulation numérique en $3 \mathrm{D}$ et plus généralement la dynamique particulière des écoulements diphasiques évoluant dans des microcanaux de section carrée est étudiée.

\section{INTRODUCTION}

The use of coflows or drippings finds his interest in various applications ( [14], [17], [11]): ink jet printing or spray atomization for example. Indeed, it is necessary to control the evolution of a diphasic jet in a view to produce droplets of different shapes and volumes. Then, the created microdroplets are often employed for their internal dynamic to mix products that are generally toxic and expensive. Since the work of Plateau and Rayleigh [16], the break-up of one liquid into another has been frequently investigated. Generally, theoretical analysis based on the concept of the linear stability method are proposed [4]. Most of the time, the studies considered inviscid cylindrical jets in air. Recently, experimental works in microfluidic show that the instability is so present when one considers diphasic flows in microchannels. In addition, several regimes of flows have been observed [9].

In this paper, we propose to analyse the complete phenomenon: the formation of the biphasic jet, the breaking of the internal phase and the creation of the microdroplet. As the breaking jet is only observable thanks to a three-dimensional modelling all numerical simulations are done in three-dimensional Cartesian meshes. Because of the size of the capillary edge used in microfluidics (about a hundred micrometres of section) and of the velocity of the flows (a few centimetre per second), the effects due to confinement and surface tension are predominant. If we look at the dimensionless numbers, generally, the Reynolds number is fewer than one and effects due to the gravity are negligible. To compute the evolution of one fluid into another one, it is required

${ }^{1}$ Université Bordeaux1, IMB - Inria Sud-Ouest 351, Cours de la Libération 33405 Talence; e-mail:bruneau@math.u-bordeaux1.fr

2 Université Bordeaux1, IMB - Inria Sud-Ouest 351, Cours de la Libération 33405 Talence; e-mail:colin@math.u-bordeaux1.fr

${ }^{3}$ Université Bordeaux1, IMB - Inria Sud-Ouest 351, Cours de la Libération 33405 Talence; e-mail:tancogne@math.u-bordeaux1.fr

(c) EDP Sciences, SMAI 2008 
to solve the Stokes equation taking into account the interfacial contribution. Following the motion of the interface between the two phases is made thanks to the Level Set method introduced by Sethian and Osher in 1987.

The article is organised as follows. The first section is dedicated to the presentation of the governing equations. The one fluid Stokes equations and the level Set method are presented. Discretizations and schemes employed are the subject of the second part. At this stage, we discuss the required stability condition when capillary effects are considered. The third part is devoted to the presentation of the numerical results.

\section{Modelling}

\subsection{Governing equations: the one fluid Stokes equations}

We consider (Figure 1) a domain $\Omega$ that contains two fluids. The two fluids, respectively called fluid one (1) and fluid two (2) occupy at each time $t$ the domains $\Omega_{1}(t)$ and $\Omega_{2}(t)$ such that $\Omega=\Omega_{1}(t) \cup \Omega_{2}(t)$. The interface $\Gamma(t)$ between the two fluids is defined as $\Gamma(t)=\bar{\Omega}_{1}(t) \cap \bar{\Omega}_{2}(t)$.

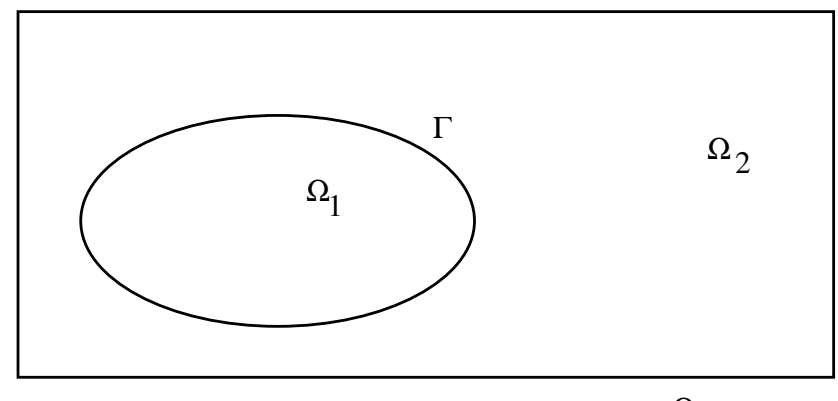

$\Omega$

FiguRE 1. Notations.

These fluids are supposed to be Newtonian, non miscible and incompressible. In the microfluidic context, the viscous effects are predominant compared to inertial ones. For this kind of flows, the Stokes equations are sufficient to represent the evolution of each fluid:

$$
\begin{cases}\nabla \cdot\left(\sigma\left(U^{1}, p^{1}\right)\right) & =0, \\ \nabla \cdot U^{1} & =0\end{cases}
$$

where $U^{1}=\left(u^{1}, v^{1}, w^{1}\right)^{t}$ is the velocity and $p^{1}$ the pressure of the fluid named fluid 1 .

$$
\begin{cases}\nabla \cdot\left(\sigma\left(U^{2}, p^{2}\right)\right) & =0, \\ \nabla \cdot U^{2} & =0,\end{cases}
$$

where $U^{2}=\left(u^{2}, v^{2}, w^{2}\right)^{t}$ is the velocity and $p^{2}$ the pressure of the fluid named fluid 2 .

$\sigma(U, p)$ denotes the stress tensor:

$$
\sigma(U, p)=2 \eta D(U)-p I d
$$

with $\eta$ the viscosity and $D(U)$ the deformation rate tensor given by $D(U)=\frac{\nabla U+(\nabla U)^{T}}{2}$.

At the interface $\Gamma$, the two fluids are non miscible and the velocity is continuous

$$
[U]=0 .
$$


The equilibrium of the normal stress is governed by the contribution of the surface tension:

$$
[\sigma(U, p) \mathbf{n}]=\gamma \kappa \mathbf{n}
$$

where $\gamma$ is the surface tension, $\mathbf{n}$ the unit normal to the interface and $\kappa$ the average curvature.

Using the distribution theory, system ( 1 - 4) can be reduced to the one fluid formulation

$$
\left\{\begin{array}{l}
\nabla \cdot(2 \eta D(U))=\nabla P+\gamma \kappa \delta_{\Gamma} \mathbf{n}, \\
\nabla \cdot U=0,
\end{array}\right.
$$

where $\delta_{\Gamma}$ is the Dirac mass on $\Gamma$ defined by

$$
\forall h \in C_{0}^{0}(\Omega), \quad<\delta_{\Gamma}, h>=\int_{\Gamma} h(x) d \sigma
$$

where $\sigma$ is the canonical surface measure and $\eta$ is the dynamic viscosity such that

$$
\eta= \begin{cases}\eta_{1} & \text { in flow } 1 \\ \eta_{2} & \text { in flow } 2\end{cases}
$$

Some boundary conditions on the velocity $U$ close the system (5). Typically, the no-slip boundary condition is imposed at the wall. The in-out flow boundary conditions are discussed when the numerical results are presented.

\subsection{Parametrization of the interface: the Level-Set method}

To follow the evolution of the interface, the Level Set method is employed. This technique was proposed by Osher and Sethian (1987). Before, the methods frequently used (Lagrangian or VOF methods) had two main drawbacks: some of them did not take precisely into account the topological changes and generally, it is difficult to compute the curvature. The Level Set formulation is based on a smooth function that gives the position of the interface and the distance of each point to this interface. The advantage of such a method is that quantities like the curvature are well computed. This point is very important for microfluidics simulations since capillary effects are predominant. It is associated to an advection equation in order to move the front: the interface between the two fluids evolves with the fluid velocity.

Thus, the interface is modelled by a level function $\phi(t, x, y, z)$. At the initial time, $\phi$ is zero at the interface, negative in one phase and positive in the other:

$$
\phi(0, x, y, z)= \begin{cases}<0 & \text { in flow } 1 \\ >0 & \text { in flow } 2 \\ 0 & \text { at the interface } \Gamma\end{cases}
$$

Its motion is governed by an advection equation

$$
\begin{cases}\partial_{t} \phi+(U . \nabla) \phi=0 & \\ \phi(0, x, y, z)=0 & \text { on } \Gamma .\end{cases}
$$

Such a modelling forces the properties of the Level Set function to be respected at each time step. In particular, the fact that the interface is represented by the zero value of the function $\phi$ :

$$
\forall t \geq 0, \quad \Gamma(t)=\{(x, y, z), \phi(t, x, y, z)=0\}
$$


When $\phi$ is known, the unit normal $\mathbf{n}$ at the interface and the curvature $\kappa$ are computed as follows,

$$
\mathbf{n}=\left.\frac{\nabla \phi}{|\nabla \phi|}\right|_{\phi=0} \text { and } \kappa=\left.\nabla \cdot\left(\frac{\nabla \phi}{|\nabla \phi|}\right)\right|_{\phi=0} .
$$

\section{Discretization of the Equations}

Now, we proceed to the discretization of the equations introduced in the previous section.

\subsection{The advection equation}

The time discretization of the advection equation (9) is explicit and a classical Euler scheme is used:

$$
\phi^{n+1}=\phi^{n}-\Delta t\left(U^{n} \cdot \nabla\right) \phi^{n}
$$

with $\Delta t$ the time step, $n+1$ the new iteration at $t^{n+1}=(n+1) \Delta t$. This choice is associated to a restriction on the time step (CFL condition) in order to ensure the stability and so the convergence:

$$
\Delta t_{c f l}<\frac{\Delta x}{\max (|U|)}
$$

The space discretization is made with a five order WENO scheme. Based on the ENO scheme proposed by Harten and al. (1987), the WENO schemes were first developed by Li and al. in order to increase the precision of ENO scheme. They proposed the fourth order WENO scheme. The idea was to make a convex combination of all possible stencils instead of taking just one. Each one is equipped with a weight that determines his contribution. In 1996, Jiang and al. [13] modify and improve the fourth order scheme to obtain a fifth order one. The use of a such high order scheme is necessary. In fact, during the numerical simulations, small structures which movements are governed by the surface tension are followed. So, it is required to be as well precise as possible and so to avoid the loss of mass.

\subsection{The hydrodynamic part}

The discretization of the incompressible Stokes equations is classical. The finite volume method on structured staggered grids is considered (Patankar, 1980). The idea is to associate different control volumes grids to the different unknowns [5]. Each unknown is located at the centers of the elements of the associated meshes. In our Cartesian three-dimensional case, the meshes consist in cubes. A typical grid is composed by a main grid associated to the pressure and three secondary grids associated to the three components of the velocity. The three Stokes equations are associated to a component of the velocity and then integrated over the considered control volumes. The continuity equation is integrated over the control volumes of the pressure. Although, the Stokes equation is a stationary equation, the time discretization of the capillary unknowns is explicit, the following scheme is employed:

$$
\begin{array}{ccc}
\nabla \cdot\left(2 \eta^{n} D\left(U^{n+1}\right)\right) & = & \nabla P^{n+1}+\gamma \kappa^{n} \delta\left(\phi^{n}\right) \nabla \phi^{n}, \\
\nabla \cdot U^{n+1} & = & 0 .
\end{array}
$$

This explicit choice introduces an usual restriction on the time step. This point is the subject of the next subsection.

The whole algorithm is the following:

(1) Initial data: at $t^{n}$ the known variables are $U^{n}, P^{n}, \eta^{n}$ and $\phi^{n}$. 
(2) Computation of $U^{n+1}$ and $P^{n+1}$ : The Augmented Lagrangian method [6] is used. This algorithm presents numerous advantages: the ease of the implementation, the reduction of the size of the linear system and an improvement of the shape of the matrix.

(a) We assume $V_{U}=U^{n}$ and $V_{P}=P^{n}$ and $V_{U}^{k}, V_{P}^{k}$ denote the intermediate variable with $k$ the Lagrangian step.

(b) Then, we solve the following linear system until the convergence criterion is achieved.

$$
\begin{aligned}
\nabla \cdot\left(2 \eta^{n} D\left(V_{U}^{k+1}\right)\right)+r \nabla\left(\nabla \cdot V_{U}^{k+1}\right) & =\nabla V_{P}^{k}+\gamma \kappa^{n} \delta\left(\phi^{n}\right) \nabla \phi^{n}, \\
V_{P}^{k+1} & =\quad V_{P}^{k}-s\left(\nabla \cdot V_{U}^{k+1}\right),
\end{aligned}
$$

with $r, s$ two fixed parameters. They are fixed here to $1(r=s=1)$.

(c) When the following criterion is achieved: $\nabla \cdot V_{U}^{k+1}<\epsilon$ (with $\epsilon \approx 1 . e^{-04}$ ), we set

$$
U^{n+1}=V_{U}^{k+1}, P^{n+1}=V_{P}^{k+1}
$$

(3) Computation of $\phi^{n+1}$

The Level Set function is moved (the discretization is made thanks to the Weno5 scheme)

$$
\phi^{n+1}=\phi^{n}-\Delta t U^{n+1}\left(\nabla \phi^{n}\right) .
$$

\section{(4) Updating of the viscosity $\eta$}

$$
\eta^{n+1}=\eta_{1}+\left(\eta_{2}-\eta_{1}\right) H\left(\phi^{n+1}\right)
$$

with $H$ the Heaviside distribution defined as

$$
H(x)= \begin{cases}1 & \text { if } x>0 \\ 0 & \text { if } x \leq 0\end{cases}
$$

\subsection{A restrictive stability condition}

In the Stokes Equation, the explicit treatment of the term associated to the surface tension required a stability criterion to maintain the convergence of the method. Commonly, the criterion proposed by Brackbill, Kote and Zemach is used [1]:

$$
\Delta t=\sqrt{\frac{1 / 2\left(\rho_{1}+\rho_{2}\right)(\Delta x)^{3}}{2 \pi \gamma}},
$$

where $\rho_{1}, \rho_{2}$ are the densities of the fluids. However, it turns out that for the microfluidics applications, this condition becomes very restrictive. Indeed, the space step are generally about $10^{-6} \mathrm{~m}$. As a consequence, the time step takes values close to $10^{-8} \mathrm{~s}$.

The derivation of the Brackbill condition is based on the study of the contribution of the instationary and inertial terms. The contribution of the densities of the two fluids in the condition (17) is a consequence of the analysis. Recently, a less restrictive stability condition was proposed by Galusinski and Vigneaux [7]:

$$
\Delta t_{\gamma} \leq c 2 \frac{\min \left(\eta_{1}, \eta_{2}\right)}{\gamma} \Delta x
$$

where $c 2$ is a constant that does not depend on the discrete parameters. This new condition takes into account the characteristics of the microfluidics flows. Indeed, the flows belong to the low Reynolds hydrodynamics and the shape of the interface is generally quickly obtained. The derivation of the new condition is based on the 
estimation of the velocity induced by a small perturbation of the interface. If the displacement of the interface is high, the perturbation will be increased and will oscillate around the interface. The condition [7] assumes that if the displacement of the interface is lower than the amplitude of the perturbation then the oscillations are deleted. Finally, in the numerical computations, the time step $\Delta t$ is chosen as being the minimum between the classical CFL condition and the above condition due to the explicit treatment of the surface tension term:

$$
\Delta t=\min \left(\Delta t_{c f l}, \Delta t_{\gamma}\right)
$$

\section{NumericAl RESUlts}

\subsection{The experimental configuration}

Experimentally, the jet is generated with a cylindrical capillary centred in a square capillary (Figure 2). Two flows are considered, we call them the internal flow and the external flow. The internal flow is injected thanks to the cylindrical capillary and the external one thanks to the square capillary. Knowing the characteristics of each flow (viscosity and surface tension coefficient), the flows rates are fixed in a way to create droplets of different sizes or to generate stable jets as in [9].

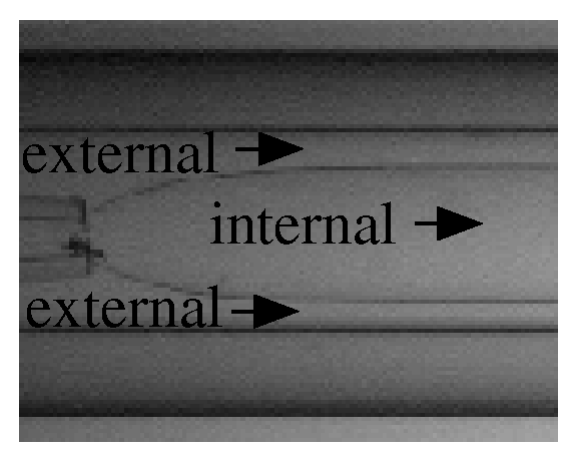

FIGURE 2. Experimental configuration: cutting of a representative configuration of two non miscible flows named internal and external.

\subsection{Inflow conditions}

The numerical configuration reproduces the experimental one.

The two flows are supposed to be injected separately. In a three-dimensional configuration, the profile for the internal flow is analytically known since it is a parabolic type in a cylindrical channel (a classical Poiseuille flow). However, the external profile is a little more complicated. First, the corresponding domain is defined on the Cartesian mesh by penalizing the injector in the square section (we noted that the more the meshing is fine, the more the representation of the injector is precise). Then, we have to compute the velocity profile numerically resolving a simplified Stokes equations since the flow is supposed to be unidirectional [12].

A typical profile employed to simulate the injection of the two flows is proposed in Figure 3. The internal and external flows rates take the following values: $Q_{e}=4500 \mu \mathrm{L} / \mathrm{h}$ and $Q_{i}=2500 \mu \mathrm{L} / \mathrm{h}$, the external microchannel has a square section $S_{c}=500 \mu \mathrm{m}$ and the radius of the cylindrical tube is $R_{i}=100 \mu \mathrm{m}$. The outlet condition is obtained with a similar method than the one proposed in [2] and [3]. It consists to use at each time step the solution obtained at the previous time step. 


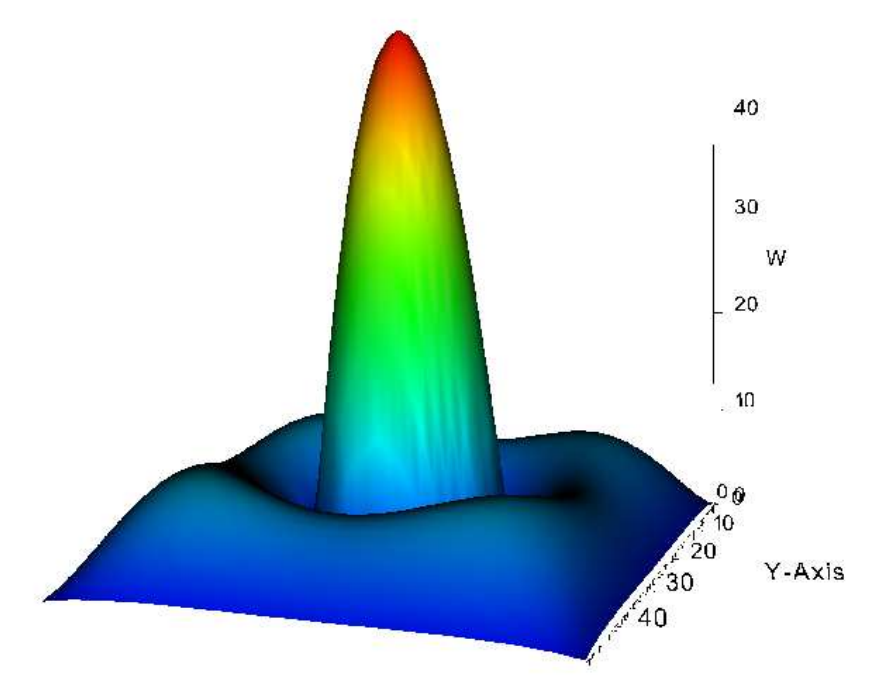

Figure 3. Typical profile employed to simulate the experimental configuration of coaxial cylinders.

\subsection{The breaking jet phenomenon}

The break-up of a jet is a complex phenomenon that refers to a well-known instability: the Rayleigh-Plateau instability. An accurate study of the hydrodynamical mechanism is now supplied.

The configuration studied is the same that the one presented in the experimental works [10]. The external microchannel has a square section $S_{c}=550 \mu \mathrm{m}$ and the radius of the cylindrical tube is $R_{i}=105 \mu \mathrm{m}$. The inner fluid is a mixture of water and glycerin of viscosity $\eta_{i}=55 \mathrm{mPa} . \mathrm{s}$ and the outer one is oil of silicone of viscosity $\eta_{e}=235 \mathrm{mPa}$.s. The surface tension between the two liquids is $\gamma=24 \mathrm{mN} / \mathrm{m}$. The Figure 4 describes the break of the inner flow and the creation of a micro droplet. The process is represented for four different times. The internal and external flows rates take the following values: $Q_{e}=5500 \mu L / h$ and $Q_{i}=2500 \mu L / h$. At $t_{1}=t_{\text {ini }} m s$ ( $t_{\text {ini }}$ corresponds to the reference time), the inner flow evolves in the outer one creating a smooth jet. At $t_{2}=t_{1}+9.5 \mathrm{~ms}$, the inner flow has reached a critical length and is pinched by the external one. Let us note that when the inner flow is tightened, the velocity fields are higher precisely at this location. Around $t_{3}=t_{2}+1.0 \mathrm{~ms}$, the created droplet and the rest of the jet are just connected by a tiny filament. Finally, at $t_{4}=t_{3}+1.2 \mathrm{~ms}$ the filament breaks, we then observe the formation of a droplet with a smooth shape due to the surface tension. When the break-up happens, the droplet is accelerated and the jet retracts to take a more spherical shape.

The Figure 5 shows a numerical result obtained with the same data in a longer microchannel. In this case, several droplets are successively generated. We show that the process is periodical and the droplets are smoothed down because of the injection of the continuous phase as shown in the two-dimensional slice presented in Figure 6 . 

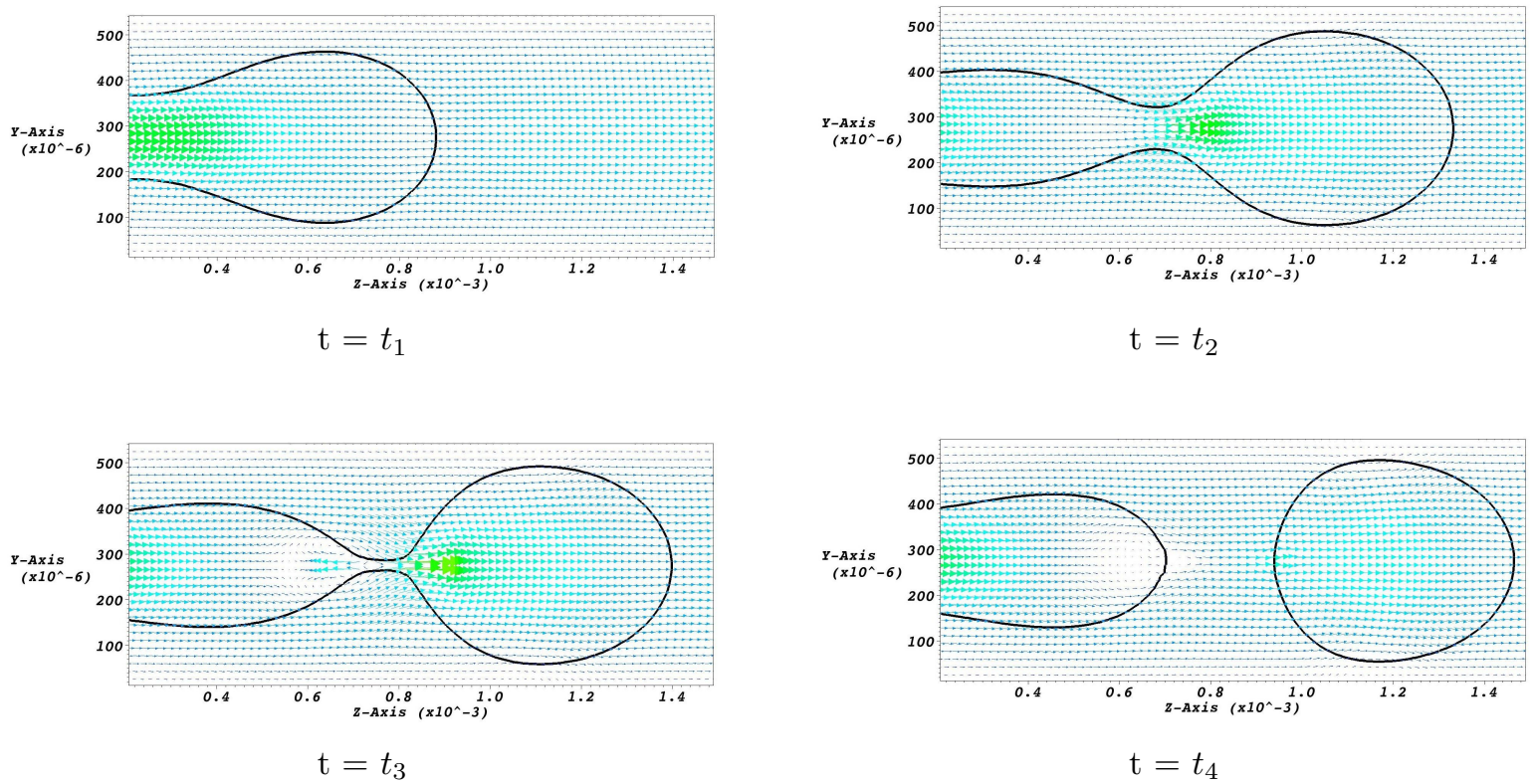

Figure 4. Formation of a droplet in a square channel when $Q_{e}=5500 \mu L / h$ and $Q_{i}=$ $2500 \mu \mathrm{L} / \mathrm{h}$. Representation of four bidimensional views, in the middle plane of the flow direction, at four different times.

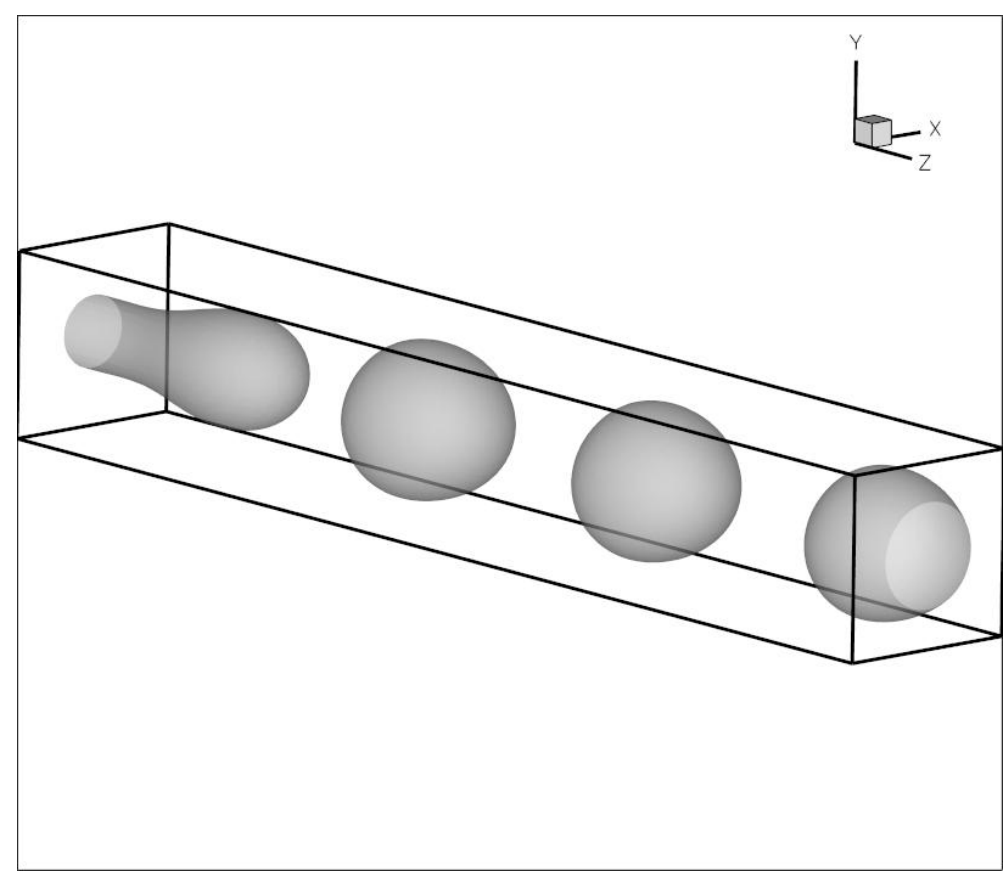

Figure 5. Succession of droplets in a microchannel when $Q_{e}=5500 \mu L / h$ and $Q_{i}=2500 \mu L / h$. 


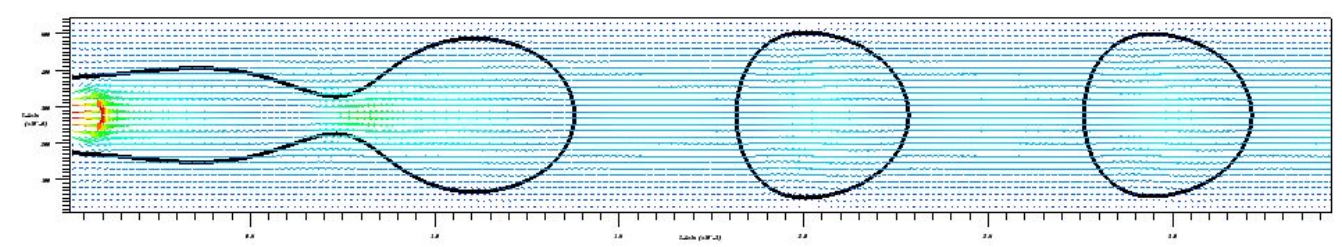

Figure 6. Shape of the droplets in a microchannel when $Q_{e}=5500 \mu L / h$ and $Q_{i}=2500 \mu L / h$.

\subsection{The parametric point of view}

Experimentally, the size of the created droplets can be controlled by fixing the value of the internal and external flows rates. To illustrate this point, a second configuration is now considered. The sections of the internal and external microchannels are unchanged. The fluids are the same but the flows rates are modified in such a way that the internal flow rate decreases, so $Q_{e}=3000 \mu L / h$ and $Q_{i}=2500 \mu L / h$. With this new parameters, the microdroplets are larger than the section of the square capillary: experimentally, such a droplet is called a plug (Figure 7).

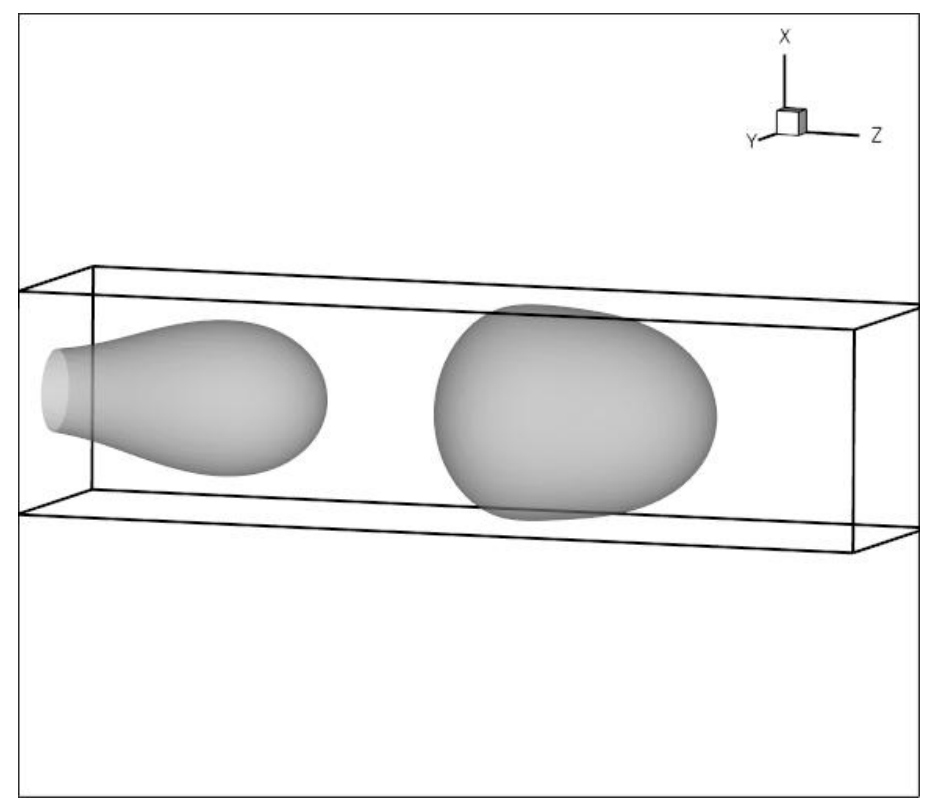

FIgURE 7. Creation of a plug in a microchannel when $Q_{e}=3000 \mu L / h$ and $Q_{i}=2500 \mu L / h$.

Now, we focus on the shape of the created plug. Two slices are proposed on Figure 8. On the left, we observed the shape of the plug in the flow direction. The plug is not ellipsoidal but smooths down at the back. Then, on the right the influence of the square section of the microchannel is underlined. We could expect that in the cross direction, more precisely at the back, the shape of the plug should be circular. But, the microdroplet takes the shape of the external capillary and presents a square shape. The comparison can be done with numerical simulations in the 3D axisymetrical case [20]. In this different configuration, the droplet is completely surrounded by the external flow and in the cross section the shape is always circular. Let us note 
that with the square capillary, the external flow can be driven only by the four corners of the micro channel.This is a typical three-dimensional effect obtained thanks to Cartesian simulations.

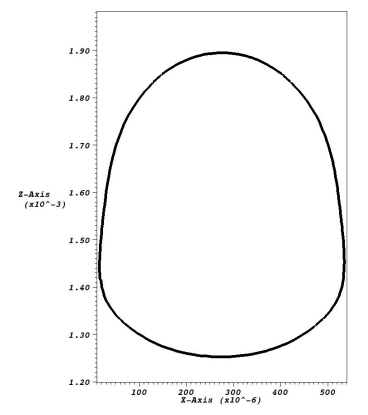

Slice in the flow direction

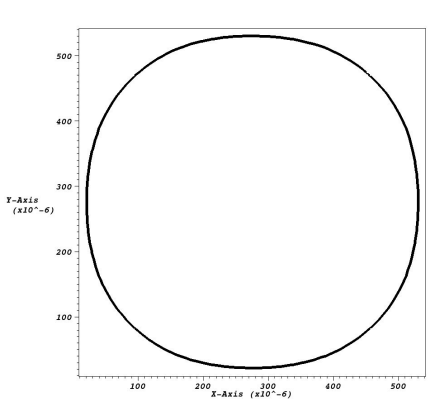

Slice in the cross section

FiguRE 8. Shape of the droplets in a microchannel when $Q_{e}=5500 \mu L / h$ and $Q_{i}=2500 \mu L / h$.

\section{Conclusions And Perspectives}

In this work, we propose three-dimensional simulations to study biphasic jet formation and break-up in a configuration where the flows are mostly governed by the pressure gradient and the surface tension. The Level Set method proposed by Osher and Sethian is used in order to follow the interface between the two phases. This method gives good results because the main quantities like the curvature and the unit normal are well computed. This study allows to analyse the breaking jet phenomenon and gives access to quantities like the pressure and the velocity of the droplet when it is created. Moreover, we can predict the shape of the droplet in the particular case when it is larger than the section of the microchannel and then compare with the case of cylindrical capillary. Exact comparisons with the experimental results are in progress. We take a special care on the representation of the internal capillary employed as an injector.

The authors wisk to thank A. Colin, J. Goyon, P. Guillot and M. Joanicot for all interesting discussions and advices concerning this work.

\section{REFERENCES}

[1] J.U. Brackbill and D.B. Kothe and C. Zemach: A Continuum Method for Modeling Surface Tension., Journal of Computational Physics, Volume 100 , Issue 2, 1992.

[2] C.-H. Bruneau and P. Fabrie: Effective downstream boundary conditions for incompressible Navier-Stokes equation, Int.J. for Num. Methods in Fluids 19, 1994.

[3] C.-H. Bruneau and P. Fabrie: New efficient boundary conditions for incompressible Navier-Stokes equation: a well-posedness result, M2AN 30, 1996.

[4] S. Chandrasekhar: Hydrodynamic and hydromagnetic stability, 1961.

[5] R. Eymard, T. Gallouet, R. Herbin: Finite Volume Methods. Handbook of Numerical Analysis, P.G Ciarlet, J.L Lions eds. (2007)

[6] M. Fortin and R. Glowinski: Augmented lagrangian methods : applications to the numerical solution of boundary-value problems, North-Holland Publishing Comp.(Studies in Mathematics and its Applications; 15), 1983.

[7] Galusinski, C.and Vigneaux, P.: Level-Set method and stability condition for curvature-driven flows. C. R. Acad. Sci. Paris, Ser. I 344(11), 703-708 (2007)

[8] P.G.de Gennes: Gouttes, bulles, perles et ondes. Edition Belin, 2005.

[9] P. Guillot and A. Colin: Stability of jet in confined pressure-driven biphasic flows at low Reynolds number, Phys. Rev. Lett. 99, $104502,2007$. 
[10] P. Guillot: Thèse de Doctorat, Ecoulement de fluides immiscibles dans un canal submillimétrique: Stabilité et application à la rhéologie, 2006.

[11] P. Guillot, P. Panizza, J.-B. Salmon, M. Joanicot, A. Colin, C.-H. Bruneau and T. Colin: Viscosimeter on a Microfluidic Chip, Langmuir, 22, 2006.

[12] E. Guyon and J.P. Hulin and L. Petit: Hydrodynamique physique. EDP sciences/CNRS Editions, 2001.

[13] G.S. Jiang and C.W Shu: Efficient Implementation of Weighted ENO Schemes. Journal of Computational Physics,126, 1996.

[14] G. Karniadakis and A. Beskok: Micro flows. Fundamentals and simulation. Springer Verlag, 2002.

[15] Osher, S., Fedkiw, R.: Level Set Methods and Dynamic Implicit Surfaces, Applied Mathematical Sciences, vol. 153. Springer (2003)

[16] Rayleigh, L., On the Instability of Jets, Proc. Lond. Math. Soc., 10, 1879.

[17] F. Sarrazin, L. Prat, G. Casamatta, M. Joanicot, C. Gourdon et G. Cristobal: Micro-drops approach in micro-reactors: mixing characterisation, La Houille Blanche, 2006.

[18] J.A. Sethian : Level Set Methods and Fast Marching Methods - Evolving interfaces in computational geometry, fluid mechanics, computer vision and materials science, Cambridge Monographs on Applied and Computational Mathematics, vol. 3, second edn. Cambridge University Press, 1999.

[19] M. Sussman, P. Smereka and S. Osher: A level set approach for computing solutions to incompressible two-phase flow. J. Comput. Phys 114, p. 146-159 (1994)

[20] P. Vigneaux: An axisymmetric Level-Set method for microdroplets hydrodynamics. under review 